\title{
Semi closed-form pricing autocallable ELS using Brownian Bridge
}

\author{
Minha Lee ${ }^{a}$, Jimin Hong ${ }^{1, b}$ \\ ${ }^{a}$ Department of Mathematics, Sungkyunkwan University, Korea; \\ ${ }^{b}$ Department of Statistics and Actuarial Science, Soongsil University, Korea
}

\begin{abstract}
This paper discusses the pricing of autocallable structured product with knock-in (KI) feature using the exit probability with the Brownian Bridge technique. The explicit pricing formula of autocallable ELS derived in the existing paper handles the part including the minimum of the Brownian motion using the inclusion-exclusion principle. This has the disadvantage that the pricing formula is complicate because of the probability with minimum value and the computational volume increases dramatically as the number of autocall chances increases. To solve this problem, we applied an efficient and robust simulation method called the Brownian Bridge technique, which provides the probability of touching the predetermined barrier when the initial and terminal values of the process following the Brownian motion in a certain interval are specified. We rewrite the existing pricing formula and provide a brief theoretical background and computational algorithm for the technique. We also provide several numerical examples computed in three different ways: explicit pricing formula, the Crude Monte Carlo simulation method and the Brownian Bridge technique.
\end{abstract}

Keywords: autocallable structured product, equity-linked security (ELS), Brownian Bridge technique

\section{Introduction}

In this paper, we introduce an alternative framework using the Brownian Bridge technique for pricing the autocallable structured product with knock-in (KI) feature. After the revision of the Securities and Exchange Law in 2003, Equity-linked security (ELS) has been one of the most popular financial derivatives and the market of ELS has been grown rapidly. As shown in Figure 1, according to Korea Financial Investment Association, issuance performance of ELS has grown from 1,526 billion KRW in 2003 to 75,073 billion KRW in 2019, about 49 times higher.

With the remarkable growth of ELS market, a lot of studies have discussed on the pricing of ELS. Among them, most studies for pricing ELS rely on the numerical analysis method such as Finite Difference Method (FDM) or Monte Carlo simulation method. Jeong et al. (2010) and Lee et al. (2020) proposed computational algorithm for two- and three-asset ELS using FDM, respectively. Kim et al. (2011) added exit-probability, which means the probability of touching barrier, to the conventional FDM. In case of Monte Carlo simulation method, Jang et al. (2019) presented numerical algorithms for one-, two-, and three-asset step-down ELS. As with many studies, these researches focus on improving accuracy and computational speed of algorithm. However, few studies discuss

\footnotetext{
${ }^{1}$ Corresponding author: Department of Statistics and Actuarial Science, Soongsil University, 369, Sangdo-Ro, DongjakGu, Seoul 06978, Republic of Korea. E-mail: jmhong@ssu.ac.kr
}

Published 31 May 2021 / journal homepage: http://csam.or.kr

(C) 2021 The Korean Statistical Society, and Korean International Statistical Society. All rights reserved. 


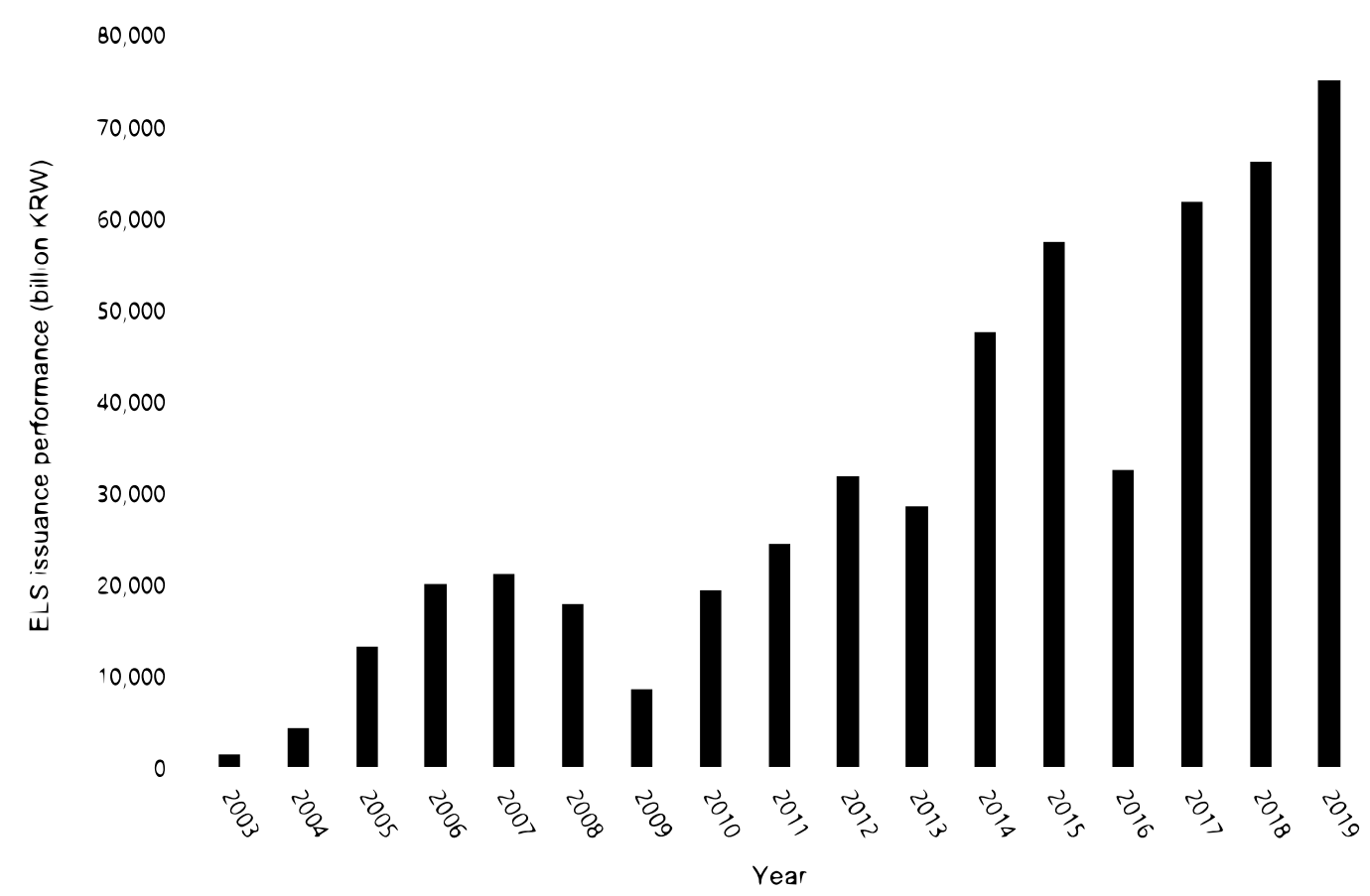

Figure 1: 2003-2019 Trend of issuance performance of ELS.

the closed-form pricing formula for ELS. Lee et al. (2019) derived a closed-form pricing formula of the product using generalized reflection principle and the inclusion-exclusion principle. The formula makes it possible to compute the exact price of the investment product. However, as can be seen later (See the Section 2), the pricing formula gets complicated and the computational volume increases dramatically as the number of autocallable chances increase which means that the number of intersections to be considered in the inclusion-exclusion principle increases. To solve this problem, we construct a practical framework to overcome these challenges by numerical method called Brownian Bridge technique (BB). In this framework, we can make the pricing formula simply and greatly compress the computational process, regardless of the number of subperiods caused by autocallable chances.

Our findings are summarized as follows: First, we rewrite the probability formula including minimum value in Lee et al. (2019) in a simplified form to solve the problem of stretched out formulas caused by the inclusion-exclusion principle. Second, we transform the formulas into conditional probabilities and apply BB to compute the conditional probabilities. Third, we verify that BB is superior to the Crude Monte Carlo method (MC) in unbiasedness, robustness, and time-efficiency. Through actual numerical test, we verify that the result using BB is closer to the actual value and less variance than the MC. In terms of computational speed, under our setting of 1,000 scenarios for both BB and $\mathrm{MC}$ and 600 monitoring steps for MC, BB is about 18 times faster than MC.

The remainder of the paper is organized as follow. Section 2 summarizes the framework of pricing autocallable structured product suggested by Lee et al. (2019). Section 3 introduces Brownian Bridge technique and its applicable algorithm. Section 4 represents numerical examples to help understanding. Finally, Section 5 concludes the paper and discusses what needs to be improved through further research. All derivation processes are provided in Appendix. 


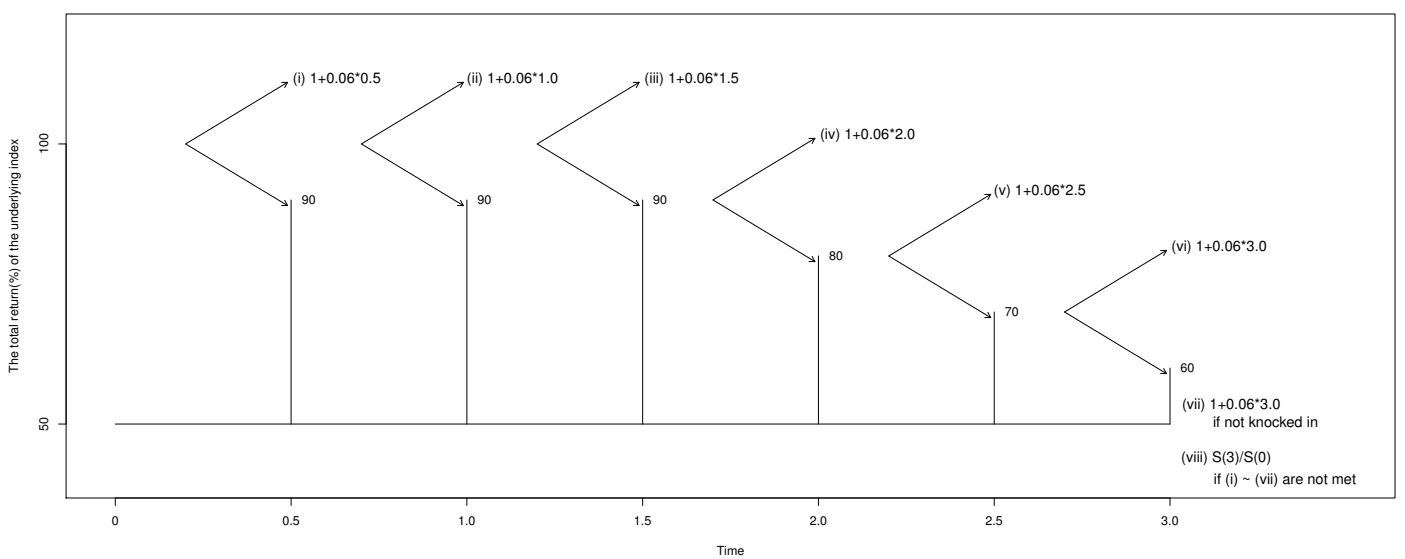

Figure 2: A popular autocallable payoff structure with 90-90-90-80-70-60/50 KI, 3 years of maturity, autocallable at every 6 months and annual coupon rate of $6 \%$. $S(t)$ denotes the price of the underlying index at time $t$ and the total return of the underlying index at time $t$ equals to $S(t) / S(0)$.

\section{Framework of pricing autocallable structured product}

The autocallable structured product with knock-in (KI) feature is one of the popular investment products which pays coupon in addition to principal if the underlying asset touches predetermined barriers. In this section, we introduce a closed-form pricing of the product derived by Lee et al. (2019). We first explain the product features through the most representative type in the Korean equity-linked security (ELS) market briefly.

With $n$ autocall thresholds and a single knock-in barrier $K_{1}-K_{2}-\cdots-K_{n} / B$ KI, here, 90-90-90-80$70-60 / 50 \mathrm{KI}$ for $n=6$, the investor has $7(6+1)$ chances to receive principal and high rate of coupon. This kind of investment product is usually called step-down KI ELS. Let us assume that the contract has a certain underlying index and offers an annualized autocallable coupon rate of $6 \%$ at every 6 months with maturity of 3 years. After 6 months from the issuance, if the underlying index is above the first autocall threshold, i.e., $90 \%$ of the initial level, the investor would receive the principal and the interest of $3 \%(6 \% \times 6$ months $)$ and the contract closed out. Otherwise, the contract proceeds to the next autocallable point and the similar process would be repeated until the maturity. Although the underlying index passes below every autocall threshold, there would be one last chance with knock-in (KI) barrier of $50 \%$. If a minimum value of the underlying index passes above $50 \%$ of its initial level, the investor would receive the principal and the interest of $18 \%(6 \% \times 3$ years $)$ at the maturity. Finally, if all 7 conditions are not met, the contract provides the actual realized rate of return at maturity by the underlying index. In this case, the investor could receive from a minimum of $0 \%$ to a maximum of $60 \%$ of principal according to index value at the maturity. Figure 2 illustrates the contract described above graphically.

Now, let us introduce the closed-form pricing formula for the autocallable product. Table 1 clearly summarizes the structure of ELS explained above. Suppose that $T$ is maturity of the contract and we divide $[0, T]$ into $n$ disjoint subperiods by $0=t_{0}<t_{i}<\cdots<t_{n}=T$. $c_{i}$ denotes the coupon rate provided to the investor at each autocallable date $t_{i}$ when the activating condition for the payoff is met. $X(t)$ is return of the underlying index at time $t, k_{i}=\ln \left(K_{i} / S(0)\right)$ and $b=\ln (B / S(0))$, which $b<k_{i}$ for all $i$ are autocall threshold and knock-in barrier in terms of yield rate, respectively, and 
Table 1: Example of structure of ELS

\begin{tabular}{cccl}
\hline \hline$i$ & Payoff & Autocallable date & \multicolumn{1}{c}{ Activating condition } \\
\hline 1 & $1+c_{1}$ & $t_{1}$ & $\operatorname{Pr}\left(X\left(t_{1}\right)>k_{1}\right)$ \\
2 & $1+c_{2}$ & $t_{2}$ & $\operatorname{Pr}\left(X\left(t_{1}\right) \leq k_{1}, X\left(t_{2}\right)>k_{2}\right)$ \\
$\vdots$ & $\vdots$ & $\vdots$ & $\vdots$ \\
6 & $1+c_{6}$ & $\operatorname{Pr}\left(\cap_{i=1}^{5}\left\{X\left(t_{i}\right) \leq k_{i}\right\}, X\left(t_{6}\right)>k_{6}\right)$ \\
7 & $1+c_{7}$ & $\operatorname{Pr}\left(\bigcap_{i=1}^{6}\left\{b<X\left(t_{i}\right) \leq k_{i}\right\}, m(0, T)>b\right)$ \\
8 & $t^{X(T)}$ & $t_{6}$ & $\operatorname{Pr}\left(\bigcap_{i=1}^{6}\left\{X\left(t_{i}\right) \leq k_{i}\right\}, m(0, T) \leq b\right)$ \\
\hline \hline
\end{tabular}

$m(0, T)=\min _{0 \leq \tau \leq T} X(\tau)$

As the activating condition met, the investor would receive principal plus interest $\left(1+c_{i}\right.$ for $i=$ $1,2, \ldots, 7)$ or realized return at each autocallable date. The pricing formula would be derived by using the elements organized in the Table 1. Section 5 in Lee et al. (2019) derives the probabilities, the expectation, and the pricing formula. Because the expressions of probabilities and the expectation in Lee et al. (2019) are too long and complicated, we re-derive the formula in a simple way. Also using the newly defined terms $P_{1}$ to $P_{8}$, we shorten the pricing formula as seen in Lemma 1 . All derivation processes are provided in Appendix A.

Lemma 1. Consider the process $\{X(t)\}$ is a Brownian motion with drift $r-\sigma^{2} / 2$ and volatility $\sigma$ under the risk-neutral measure where $r$ is the risk-free rate. Then, the probabilities and the expectation can be expressed as follows: For $b<k_{i}, i=1, \ldots 6$,

(i) Probabilities $P_{i}(i=1, \ldots, 6)$ for $i^{\text {th }}$ payoff

$$
P_{i}=\operatorname{Pr}\left(\bigcap_{j=1}^{i-1}\left\{X\left(t_{j}\right) \leq k_{j}\right\}, X\left(t_{i}\right)>k_{i}\right),
$$

(ii) Probability $P_{7}$ for $7^{\text {th }}$ payoff

$$
P_{7}=\operatorname{Pr}\left(\bigcap_{i=1}^{6}\left\{X\left(t_{i}\right) \leq k_{i}\right\}, m(0, T)>b\right)=\sum_{J \subset\{1, \ldots, 6\}}(-1)^{|J|} \operatorname{Pr}\left(\bigcap_{j \in J}\left\{X\left(t_{j}\right)>k_{j}\right\}, m(0, T)>b\right),
$$

(iii) Expectation $P_{8}$ for $8^{\text {th }}$ payoff

$$
\begin{aligned}
P_{8} & =e^{-r T} E\left[e^{X(T)} I\left(\bigcap_{i=1}^{6}\left\{X\left(t_{i}\right) \leq k_{i}\right\}, m(0, T) \leq b\right)\right] \\
& =\operatorname{Pr}^{*}\left(\bigcap_{i=1}^{6}\left\{X\left(t_{i}\right) \leq k_{i}\right\}, m(0, T) \leq b\right) \\
& =\operatorname{Pr}^{*}\left(\bigcap_{i=1}^{6}\left\{X\left(t_{i}\right) \leq k_{i}\right\}\right)-\sum_{J \subset\{1, \ldots, 6\}}(-1)^{|J|} \operatorname{Pr}^{*}\left(\bigcap_{j \in J}\left\{X\left(t_{j}\right)>k_{j}\right\}, m(0, T)>b\right),
\end{aligned}
$$


where $|J|$ denotes the cardinality of set $J$ and $\operatorname{Pr}^{*}(\cdot)$ in (2.3) denotes the probability with drift $r+\sigma^{2} / 2$. See Gerber and Shiu (1994) for more details on this. Once coupon rate, probabilities and expectation of payoff, and interest rate are set, we can calculate time-0 price of the product as follows:

$$
\begin{aligned}
\text { Price }_{0}= & \sum_{i=1}^{6} 100 e^{-r t_{i}}\left(1+c_{i}\right) \operatorname{Pr}\left(\bigcap_{j=1}^{i-1}\left\{X\left(t_{j}\right) \leq k_{j}\right\}, X\left(t_{i}\right)>k_{i}\right) \\
& +100 e^{-r T}\left(1+c_{7}\right) \operatorname{Pr}\left(\bigcap_{j=i}^{6}\left\{X\left(t_{i}\right) \leq k_{i}\right\}, m(0, T)>b\right) \\
& +e^{-r T} E\left[100 e^{X(T)} I\left(\bigcap_{i=1}^{6}\left\{X\left(t_{i}\right) \leq k_{i}\right\}, m(0, T) \leq b\right)\right] \\
= & 100\left(\sum_{i=1}^{6}\left(1+c_{i}\right) P_{i}+e^{-r T}\left(1+c_{7}\right) P_{7}+P_{8}\right) .
\end{aligned}
$$

Proof: See Appendix A.

For 6 subperiods, we can easily compute $P_{1}$ to $P_{6}$ without considering the inclusion-exclusion principle because they do not include the minimum value and many statistical packages support such functions. However, in the case of $P_{7}$ and $P_{8}$, it is difficult to find such convenient functions because these two values are only obtained by the inclusion-exclusion principle. Thus, we have to do $\sum_{i=1}^{6}\left(\begin{array}{l}6 \\ i\end{array}\right)$ computation each to obtain them. As in Figure 3, this number increases dramatically as the number of subperiods increases. For example, if the contract described earlier provides autocall chances quarterly which means 4 times a year, the number of subperiods increases from 6 into 12 . Then we will have to do more than 8,000 calculations to compute $P_{7}$ and $P_{8}$. We need another way to reduce the computational cost, and one of them is exit probability with Brownian Bridge.

\section{Brownian Bridge and autocallable ELS}

\subsection{Brownian Bridge (BB)}

We need to compute the probability and expectation which the process $X(t)$ touches the predetermined barrier during the entire contract period. This problem can be solved by using the exit probability of the $\mathrm{BB}$ process that deals with the dynamics of a Brownian motion when its initial value and terminal value in the given computational interval are known. Fortunately, there are many studies on the exit probability with one-dimensional $\mathrm{BB}$. The biggest advantage of this technique is that it is timeefficient compared to MC. In order to compute a probability precisely using MC, it is necessary to divide the computational interval as much as possible. However, with BB, we only need to know two values, the initial and the terminal value for each computational interval. In this study, we only need to know 7 values since we have 6 autocallable dates.

Another advantage of using BB is that it reduces bias caused by discretization. MC is a simulating method by discretizing the continuous flow of underlying index, so a systematic bias occurs accordingly. This is called monitoring bias or discretization bias. Systematically, in terms of pricing barrier option, price of Knock-Out option is overestimated, but price of Knock-In option is underestimated. When we divide computational interval into $M$ monitoring steps, order $O\left(M^{-1 / 2}\right)$ monitoring bias occurs. However, as BB compute exit probability explicitly using the initial and the terminal values, the 


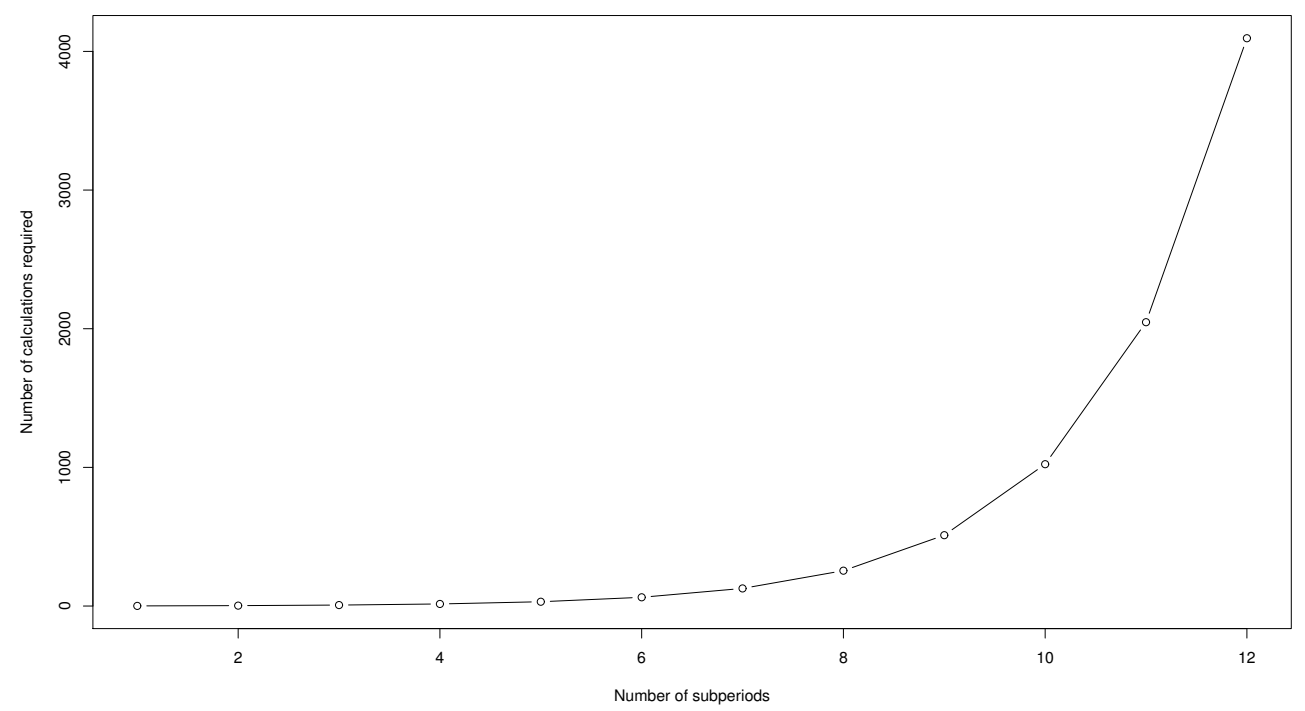

Figure 3: The number of computations required with the number of subperiods.

bias from discretization would be disappeared. Thus, for $N$ scenarios and $M$ monitoring steps, the bias of $\mathrm{MC}$ is much greater than that of $\mathrm{BB}$ in terms of cost. That is,

$$
\text { Bias of } \mathrm{MC}=O\left(M^{-\frac{1}{2}}\right)>O\left((N \times M)^{-\frac{1}{2}}\right)=\text { bias of BB. }
$$

For more details, we refer to Gobet (2009).

In this section, we introduce the explicit formula for the exit probability with BB and use it to calculate $P_{7}$ and $P_{8}$ defined above. Note that exit probability between time $s$ and $t$ means the probability that the minimum value fall below the KI barrier $b$ between time $s$ and $t, \operatorname{Pr}\left(\min _{s \leq \tau \leq t} X(\tau) \leq b\right)$, but in this paper, the probability that the minimum value pass above the barrier is mainly used for convenience of computation. And this probability is expressed as the product of the probability for each subperiod divided by autocallable date, not the probability for the entire period. This is because we consider the case where the KI barrier levels are different for each subperiod. The exit probability in the given setting can be computed as follows. Suppose that $\left\{X(\tau), \tau \in\left[t_{i-1}, t_{i}\right]\right\}$ is a one-dimensional Brownian motion with volatility $\sigma$. Assuming that we already know the initial and the terminal values of the process, this conditional process become Brownian Bridge. Let $m\left(t_{i-1}, t_{i}\right)=\min _{t_{i-1} \leq \tau \leq t_{i}} X(\tau)$, $b$ is the predetermined knock-in barrier level applied to subperiod $\left[t_{i-1}, t_{i}\right]$, and yield rate at time $t_{i-1}$ and $t_{i}$ is given as $x_{i-1}$ and $x_{i}$, respectively. Then,

$$
\operatorname{Pr}\left(m\left(t_{i-1}, t_{i}\right)>b \mid X\left(t_{i-1}\right)=x_{i-1}, X\left(t_{i}\right)=x_{i}\right)= \begin{cases}1-\exp \left\{-\frac{2\left(b-x_{i-1}\right)\left(b-x_{i}\right)}{\sigma^{2}\left(t_{i}-t_{i-1}\right)}\right\}, & \text { if } x_{i-1}>b \text { and } x_{i}>b, \\ 0, & \text { otherwise. }\end{cases}
$$

For convenience, we denote (3.1) by $p_{b}\left(x_{i-1}, x_{i}\right)$.

\subsection{Construction of $\mathrm{BB}$ algorithm}

Now, we need to transform $P_{7}$ and $P_{8}$ into a form that we can apply BB. Thus, we rewrite those two probabilities into (3.2) and (3.3) so that we can apply BB to the conditional probabilities of both equations. Following lemma shows rederived $P_{7}$ and $P_{8}$. 
Lemma 2. For $b<k_{i}, i=1, \ldots, 6, P_{7}$ and $P_{8}$ can be rederived as follows:

$$
\begin{aligned}
P_{7} & =\operatorname{Pr}\left(\bigcap_{i=1}^{6}\left\{b<X\left(t_{i}\right) \leq k_{i}\right\}, m(0, T)>b\right) \\
& =\operatorname{Pr}\left(\bigcap_{i=1}^{6}\left\{b<X\left(t_{i}\right) \leq k_{i}\right\}\right) \times E\left[\prod_{i=1}^{6} \operatorname{Pr}\left(m\left(t_{i-1}, t_{i}\right)>b \mid X\left(t_{i-1}\right), X\left(t_{i}\right)\right) \mid \bigcap_{i=1}^{6}\left\{b<X\left(t_{i}\right) \leq k_{i}\right\}\right]
\end{aligned}
$$

and

$$
\begin{aligned}
P_{8} & =\operatorname{Pr}^{*}\left(\bigcap_{i=1}^{6}\left\{X\left(t_{i}\right) \leq k_{i}\right\}, m(0, T) \leq b\right) \\
& =\operatorname{Pr}^{*}\left(\bigcap_{i=1}^{6}\left\{X\left(t_{i}\right) \leq k_{i}\right\}\right) \times\left(1-E^{*}\left[\prod_{i=1}^{6} \operatorname{Pr}^{*}\left(m\left(t_{i-1}, t_{i}\right)>b \mid X\left(t_{i-1}\right), X\left(t_{i}\right)\right) \mid \bigcap_{i=1}^{6}\left\{X\left(t_{i}\right) \leq k_{i}\right\}\right]\right),
\end{aligned}
$$

where $E^{*}[\cdot]$ denotes the expectation with drift $r+\sigma^{2} / 2$.

Proof: See Appendix B.

We would compute expectation of conditional probabilities including minimum value in (3.2) and (3.3) through BB algorithm. Both $\operatorname{Pr}\left(\bigcap_{i=1}^{6}\left\{b<X\left(t_{i}\right) \leq k_{i}\right\}\right)$ and $\operatorname{Pr}^{*}\left(\bigcap_{i=1}^{6}\left\{X\left(t_{i}\right) \leq k_{i}\right\}\right)$ in Lemma 2 can be calculated explicitly. First, based on (3.2), we could set up the following algorithm for computing $P_{7}$. Suppose the process $\{X(t)\}$ is a Brownian motion with drift $\mu=r-\sigma^{2} / 2$ and volatility $\sigma$, and $X(0)=0$. We generate $N$ number of scenarios of the underlying index paths representing various situations. For each scenario, we could generate index path by each autocallable date $t_{i}, i=1, \ldots, 6$

$$
X\left(t_{i}\right)=X\left(t_{i-1}\right)+\mu \Delta t_{i}+\sigma \sqrt{\Delta t_{i}} Z \quad \text { for } i=1,2, \ldots, 6 \text { and } Z \sim N(0,1) .
$$

After generating index paths for all scenarios, extract only scenarios that meet the condition $b<$ $x_{i} \leq k_{i}$ for all $i$ where $x_{i}$ is simulated value of $X\left(t_{i}\right)$. Using $N_{1}$ number of extracted scenarios, we can compute $P_{7}$ as follows:

$$
\hat{P}_{7}=\operatorname{Pr}\left(\bigcap_{i=1}^{6}\left\{b<X\left(t_{i}\right) \leq k_{i}\right\}\right) \times \frac{1}{N_{1}} \sum_{b<x_{i} \leq k_{i} \forall i}\left[\prod_{i=1}^{6} p_{b}\left(x_{i-1}, x_{i}\right)\right] .
$$

We can also compute $P_{8}$ in a similar way except for drift and extracting condition. In generating index paths, (3.4) is used but drift $r-\sigma^{2} / 2$ is shifted to $r+\sigma^{2} / 2$. Also, extracting condition is changed to $x_{i}^{*}<k_{i}$ where $x_{i}^{*}$ is simulated value of $X\left(t_{i}\right)$ under the shifted drift. Again, using $N_{2}$ number of extracted scenarios, we can compute $P_{8}$ as follows:

$$
\hat{P}_{8}=\operatorname{Pr}^{*}\left(\bigcap_{i=1}^{6}\left\{X\left(t_{i}\right) \leq k_{i}\right\}\right) \times\left(1-\frac{1}{N_{2}} \sum_{x_{i}^{*} \leq k_{i} \forall}\left[\prod_{i=1}^{6} p_{b}\left(x_{i-1}^{*}, x_{i}^{*}\right)\right]\right) .
$$

By following the process listed so far, we can obtain all the elements needed for pricing the autocallable ELS. $P_{1}$ through $P_{6}$ can be easily obtained by statistical packages and $P_{7}$ and $P_{8}$ can be obtained by BB algorithm explained above. Since the algorithm is a method of multiplying the probabilities obtained by simulation, the error occurring in each subperiod remains without canceling. Therefore, the results in our study are limited by the case of sufficiently small subperiod $n$. Note that if the number of $n$ become sufficiently large, this error cannot be negligible. Next, we verify the performance of our proposed algorithm through numerical analysis. 
Table 2: Numerical example of time-0 price of autocallable ELS

\begin{tabular}{|c|c|c|c|c|c|c|c|c|c|c|}
\hline \multirow{2}{*}{$r$} & \multirow{2}{*}{$c$} & \multicolumn{3}{|c|}{ Explicit Forumla } & \multicolumn{3}{|c|}{ Brownian Bridge } & \multicolumn{3}{|c|}{ Crude Monte Carlo } \\
\hline & & $\sigma=20 \%$ & $\sigma=25 \%$ & $\sigma=30 \%$ & $\sigma=20 \%$ & $\sigma=25 \%$ & $\sigma=30 \%$ & $\sigma=20 \%$ & $\sigma=25 \%$ & $\sigma=30 \%$ \\
\hline \multirow{3}{*}{$3 \%$} & $5.0 \%$ & 100.42 & 98.79 & 96.76 & 100.50 & 98.82 & 96.76 & 100.48 & 99.02 & 96.70 \\
\hline & $6.5 \%$ & 101.50 & 99.91 & 97.87 & 101.59 & 99.94 & 97.88 & 101.57 & 100.15 & 97.82 \\
\hline & $8.0 \%$ & 102.59 & 101.03 & 98.99 & 102.68 & 101.06 & 98.99 & 102.66 & 101.27 & 98.93 \\
\hline \multirow{3}{*}{$4 \%$} & $5.0 \%$ & 99.81 & 98.28 & 96.33 & 99.81 & 98.35 & 96.26 & 99.84 & 98.55 & 96.41 \\
\hline & $6.5 \%$ & 100.86 & 99.38 & 97.43 & 100.86 & 99.44 & 97.36 & 100.89 & 99.66 & 97.52 \\
\hline & $8.0 \%$ & 101.92 & 100.47 & 98.53 & 101.92 & 100.54 & 98.45 & 101.95 & 100.77 & 98.62 \\
\hline \multirow{3}{*}{$5 \%$} & $5.0 \%$ & 99.21 & 97.77 & 95.89 & 99.15 & 97.85 & 95.79 & 99.28 & 97.97 & 96.06 \\
\hline & $6.5 \%$ & 100.24 & 98.85 & 96.98 & 100.18 & 98.92 & 96.87 & 100.31 & 99.05 & 97.14 \\
\hline & $8.0 \%$ & 101.26 & 99.92 & 98.06 & 101.21 & 100.00 & 97.95 & 101.34 & 100.13 & 98.23 \\
\hline
\end{tabular}

Table 3: Numerical example of breakeven coupon rate $C^{*}$

\begin{tabular}{|c|c|c|c|c|c|c|c|c|c|}
\hline \multirow{2}{*}{$r$} & \multicolumn{3}{|c|}{ Explicit Forumla } & \multicolumn{3}{|c|}{ Brownian Bridge } & \multicolumn{3}{|c|}{ Crude Monte Carlo } \\
\hline & $\sigma=20 \%$ & $\sigma=25 \%$ & $\sigma=30 \%$ & $\sigma=20 \%$ & $\sigma=25 \%$ & $\sigma=30 \%$ & $\sigma=20 \%$ & $\sigma=25 \%$ & $\sigma=30 \%$ \\
\hline $3 \%$ & $4.42 \%$ & $6.62 \%$ & $9.36 \%$ & $4.30 \%$ & $6.58 \%$ & $9.36 \%$ & $4.33 \%$ & $6.31 \%$ & $9.44 \%$ \\
\hline $4 \%$ & $5.27 \%$ & $7.35 \%$ & $10.01 \%$ & $5.28 \%$ & $7.26 \%$ & $10.11 \%$ & $5.23 \%$ & $6.96 \%$ & $9.88 \%$ \\
\hline $5 \%$ & $6.15 \%$ & $8.11 \%$ & $10.69 \%$ & $6.24 \%$ & $8.00 \%$ & $10.85 \%$ & $6.05 \%$ & $7.82 \%$ & $10.45 \%$ \\
\hline
\end{tabular}

\section{Numerical analysis}

In this section, we provide numerical examples of the time- 0 price and breakeven coupon rates of autocallable ELS using three different methods, which is the explicit formula, BB and MC. To verify the superiority of $\mathrm{BB}$, we also provide the results of relative error and some summary statistics of the distributions of $P_{7}$ and $P_{8}$ compared to MC. In this simulation, we generate 10,000 scenarios for both $\mathrm{BB}$ and $\mathrm{MC}$. In the case of $\mathrm{MC}$, the entire contract period is divided into 600 monitoring steps.

In Tables 2 and 3, we summarize the results of numerical examples of the time- 0 price and breakeven coupon rates of autocallable ELS. Column "Explicit formula" denotes the results computed by the explicit pricing formula derived in Lee et al. (2019), and column "Brownian Bridge" and "Crude Monte Carlo" are the results computed using each simulation method, respectively. We assume an autocallable ELS with an initial underlying index of 100, autocallable date at every 6 months during 3 years of maturity. The autocall thresholds are given as 90-90-90-80-70-60 / 50 KI. The coupon rates given to the investor are set proportional to the autocall time for certain rate $C$, i.e., $c_{1}=0.5 \times C, c_{2}=1 \times C, \ldots, c_{6}=3 \times C$, and $c_{7}=3 \times C$. In Table 2, time-0 price of autocallable ELS is directly proportional to $C$, and inversely proportional to the risk-free rate and volatility of underlying index.

As shown in Table 2, while the result of BB is somewhat larger or smaller than the result obtained by explicit formula, it can be seen that most of the result obtained by $\mathrm{MC}$ are larger. This seems to be the result of discretization bias, the problem of $\mathrm{MC}$ mentioned in Section 3. In the case of the $7^{\text {th }}$ payoff, the present value is the largest, but since it has the same form as the knock-out barrier option, the activating probability in the MC can be overestminated by discretization. As a result, in most cases, the results of MC are larger than other results.

Also, by setting the time-0 price equals to initial level of underlying index, we can obtain the breakeven point of the coupon rates $C^{*}$. Suppose that the risk-free rate and $P_{i}$ for $i=1,2, \ldots, 8$ are 
Table 4: Numerical examples of relative error of the time-0 price for autocallable ELS

\begin{tabular}{|c|c|c|c|c|c|c|c|}
\hline \multirow{2}{*}{$r$} & \multirow{2}{*}{$c$} & \multicolumn{3}{|c|}{ Brownian Bridge } & \multicolumn{3}{|c|}{ Crude Monte Carlo } \\
\hline & & $\sigma=20 \%$ & $\sigma=25 \%$ & $\sigma=30 \%$ & $\sigma=20 \%$ & $\sigma=25 \%$ & $\sigma=30 \%$ \\
\hline \multirow{3}{*}{$3 \%$} & $5.0 \%$ & $0.08 \%$ & $0.03 \%$ & $0.00 \%$ & $0.06 \%$ & $0.23 \%$ & $0.06 \%$ \\
\hline & $6.5 \%$ & $0.08 \%$ & $0.03 \%$ & $0.00 \%$ & $0.06 \%$ & $0.24 \%$ & $0.06 \%$ \\
\hline & $8.0 \%$ & $0.08 \%$ & $0.03 \%$ & $0.00 \%$ & $0.06 \%$ & $0.24 \%$ & $0.06 \%$ \\
\hline \multirow{3}{*}{$4 \%$} & $5.0 \%$ & $0.00 \%$ & $0.07 \%$ & $0.07 \%$ & $0.03 \%$ & $0.28 \%$ & $0.09 \%$ \\
\hline & $6.5 \%$ & $0.00 \%$ & $0.07 \%$ & $0.07 \%$ & $0.03 \%$ & $0.29 \%$ & $0.09 \%$ \\
\hline & $8.0 \%$ & $0.00 \%$ & $0.07 \%$ & $0.07 \%$ & $0.03 \%$ & $0.29 \%$ & $0.09 \%$ \\
\hline \multirow{3}{*}{$5 \%$} & $5.0 \%$ & $0.06 \%$ & $0.08 \%$ & $0.11 \%$ & $0.07 \%$ & $0.20 \%$ & $0.17 \%$ \\
\hline & $6.5 \%$ & $0.06 \%$ & $0.08 \%$ & $0.11 \%$ & $0.07 \%$ & $0.21 \%$ & $0.17 \%$ \\
\hline & $8.0 \%$ & $0.06 \%$ & $0.08 \%$ & $0.11 \%$ & $0.07 \%$ & $0.21 \%$ & $0.17 \%$ \\
\hline
\end{tabular}

Table 5: Numerical example of relative error of breakeven coupon rate

\begin{tabular}{|c|c|c|c|c|c|c|}
\hline \multirow{2}{*}{$r$} & \multicolumn{3}{|c|}{ Brownian Bridge } & \multicolumn{3}{|c|}{ Crude Monte Carlo } \\
\hline & $\sigma=20 \%$ & $\sigma=25 \%$ & $\sigma=30 \%$ & $\sigma=20 \%$ & $\sigma=25 \%$ & $\sigma=30 \%$ \\
\hline $3 \%$ & $2.64 \%$ & $0.61 \%$ & $0.05 \%$ & $1.98 \%$ & $4.76 \%$ & $0.80 \%$ \\
\hline $4 \%$ & $0.02 \%$ & $1.24 \%$ & $0.98 \%$ & $0.80 \%$ & $5.33 \%$ & $1.32 \%$ \\
\hline $5 \%$ & $1.33 \%$ & $1.34 \%$ & $1.51 \%$ & $1.76 \%$ & $3.62 \%$ & $2.24 \%$ \\
\hline
\end{tabular}

given, then the breakeven coupon rate can be obtained as follows:

$$
\begin{aligned}
& 100\left(\sum_{i=1}^{6} e^{-r t_{i}}\left(1+c_{i}^{*}\right) P_{i}+e^{-r t_{6}}\left(1+c_{7}^{*}\right) P_{7}+P_{8}\right) \\
& =100\left(C^{*}\left(\sum_{i=1}^{6} e^{-r t_{i}} t_{i} P_{i}+e^{-r t_{6}} t_{6} P_{7}\right)+\sum_{i=1}^{6} e^{-r t_{i}} P_{i}+e^{-r t_{6}} P_{7}+P_{8}\right)=100, \\
& \therefore C^{*}=\frac{1-\left(\sum_{i=1}^{6} e^{-r t_{i}} P_{i}+e^{-r t_{6}} P_{7}+P_{8}\right)}{\sum_{i=1}^{6} e^{-r t_{i}} t_{i} P_{i}+e^{-r t_{6}} t_{6} P_{7}},
\end{aligned}
$$

where $c_{i}^{*}=t_{i} \times C^{*}$. Table 3 shows the breakeven coupon rates computed by (4.1). Contrary to the time- 0 prices, the breakeven coupon rate is directly proportional to the risk-free rate and the volatility. Also, if $C$ in Table 2 is bigger than $C^{*}$ under the same parameter values, the time- 0 price is greater than 100 and vice versa.

In Tables 2 and 3, we can achieve similar results to explicit formula through BB. To clearly show the usefulness of the technique, the relative error with the actual value is presented. In addition, to show the robustness of the technique, we compare it to MC. When $V_{E}$ is exact value and $V_{A}$ is approximated value, the relative error is obtained as

$$
\text { Relative error }=\left|\frac{V_{E}-V_{A}}{V_{E}}\right| .
$$

Tables 4 and 5 show the relative errors of the two simulation methods for the time- 0 price and the breakeven coupon rate, respectively. In some cases, the error of BB is larger than that of MC, but in most cases, the error of $\mathrm{MC}$ is much larger.

Finally, given $r=3 \%$ and $\sigma=20 \%, 100$ values of $P_{7}$ and $P_{8}$ were generated by each method to create distribution. In Figure 4, panel (4a) shows the distribution of approximated value of $P_{7}$ and 


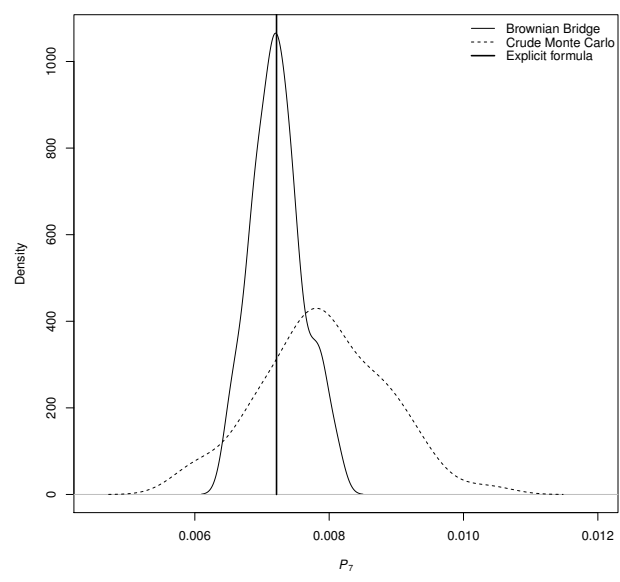

(a) Distribution of $P_{7}$

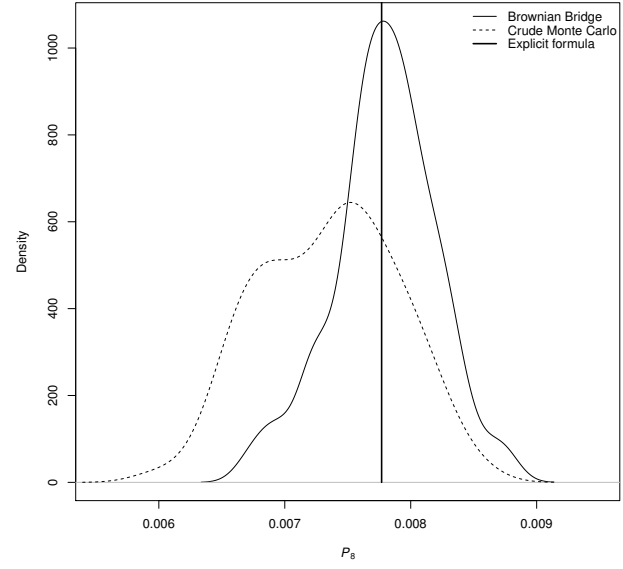

(b) Distribution of $P_{8}$

Figure 4: Distribution of simulated values of $P_{7}$ and $P_{8}$. The values of $P_{7}$ and $P_{8}$ obtained by the explicit formula are $0.72 \%$ and $0.78 \%$, respectively.

Table 6: Summary description of the distribution of $P_{7}$ and $P_{8}$ computed by Brownian Bridge and Crude Monte Carlo method

\begin{tabular}{|c|c|c|c|c|c|c|}
\hline \multirow{2}{*}{ Description } & \multicolumn{2}{|c|}{$P_{7}$} & \multicolumn{2}{|c|}{$P_{8}$} & \multicolumn{2}{|c|}{ Computational time } \\
\hline & $\mathrm{BB}$ & $\mathrm{MC}$ & $\mathrm{BB}$ & $\mathrm{MC}$ & $\mathrm{BB}$ & $\mathrm{MC}$ \\
\hline Min. & $0.65 \%$ & $0.57 \%$ & $0.67 \%$ & $0.60 \%$ & $44.36 \mathrm{sec}$. & $811.78 \mathrm{sec}$. \\
\hline Mean & $0.72 \%$ & $0.79 \%$ & $0.78 \%$ & $0.73 \%$ & $45.20 \mathrm{sec}$. & $833.70 \mathrm{sec}$. \\
\hline Max. & $0.81 \%$ & $1.05 \%$ & $0.88 \%$ & $0.86 \%$ & $56.95 \mathrm{sec}$. & $1181.31 \mathrm{sec}$. \\
\hline St.Dv & $0.04 \%$ & $0.09 \%$ & $0.04 \%$ & $0.05 \%$ & $1.64 \mathrm{sec}$. & $49.71 \mathrm{sec}$ \\
\hline
\end{tabular}

panel (4b) shows the distribution of approximated value of $P_{8}$. Solid lines, dash lines, and thick lines in Figure 4 represent the results from Brownian Bridge technique, the Crude Monte Carlo method, and the explicit formula, respectively. Although it is not perfectly symmetrical, the results are distributed around the actual values. And the important thing is that the results of $\mathrm{MC}$ are more widely distributed than that of BB. That means BB is more robust than MC.

The robustness of $\mathrm{BB}$ can also be conformed through the summary statistics of the simulation values in Table 6 . The ranges (= Max. - Min.) of the probabilities obtained by MC are wider than what obtained by BB. In the case of standard deviation, MC is also much larger than the technique. In addition, we check the time-spent creating the distribution to show that BB is a time-efficient method compared to MC. As you can see, in terms of mean, computing with BB takes about $5.42 \%$ of the time compared to MC. That is, BB is about 18 times more time-efficient than MC. Considering that the maturity of step-down KI ELS is mostly 2-3 years, no matter how finely autocall change is subdivided, for example, once a month, there is a limitation to which the size $\mathrm{n}$ of the subperiod can be increased. Thus, this computational efficiency is expected to be maintained.

\section{Conclusion}

In this paper, we illustrate how to obtain the price of autocallable structured product with KI feature using conditional probabilities and the Brownian Bridge technique. The conditional probabilities 
make the pricing formula simpler than the old one and the technique makes it possible to compute the probabilities quickly and accurately. Also, the technique is far superior to the Crude Monte Carlo method in terms of accuracy, robustness, and computational speed.

We focus on pricing the product with one underlying index. However, as for the market shares of issuance by the number of underlying assets, are one-, two-, three-, and more than four-asset account for $3.90 \%, 3.08 \%, 88.83 \%$, and $4.19 \%$, respectively. Future research could examine the pricing frameworks for generalized investment product such as increasing the number of underlying index or diversifying knock-in barrier types to meet the diverse needs of derivatives investors.

To meet the diverse needs of derivatives investors, pricing frameworks for generalized investment product such as increasing the number of underlying index or diversifying knock-in barrier types are needed.

\section{Appendix A: Proof of (2.2) and (2.3) in Lemma 1}

Proof of (2.2): For $b<k_{i}, i=1, \ldots, 6$,

$$
P_{7}=\operatorname{Pr}\left(\bigcap_{i=1}^{6}\left\{b<X\left(t_{i}\right) \leq k_{i}\right\}, m(0, T)>b\right)=\operatorname{Pr}\left(\left(\bigcup_{i=1}^{6}\left\{X\left(t_{i}\right)>k_{i}\right\}\right)^{c}, m(0, T)>b\right) .
$$

With property of complement set, (A.1) can be rewritten as

$$
\operatorname{Pr}(m(0, T)>b)-\operatorname{Pr}\left(\bigcup_{i=1}^{6}\left\{X\left(t_{i}\right)>k_{i}\right\}, m(0, T)>b\right) .
$$

Also, using the inclusive-exclusive principle, we can decompose the union in (A.2) and the decomposed probabilities are simplified as

$$
\sum_{J \subset\{1, \ldots, 6\}}(-1)^{|J|} \operatorname{Pr}\left(\bigcap_{j \in J}\left\{X\left(t_{i}\right)>k_{i}\right\}, m(0, T)>b\right) .
$$

See Lee et al. (2019) for computing probabilities including minimum value $m(0, T)$.

Proof of (2.3): For $b<k_{i}, i=1, \ldots, 6$,

By using factorization formula, we can obtain

$$
\begin{aligned}
P_{8} & =e^{-r T} E\left[e^{X(T)} I\left(\bigcap_{i=1}^{6}\left\{X\left(t_{i}\right) \leq k_{i}\right\}, m(0, T) \leq b\right)\right] \\
& =\operatorname{Pr}^{*}\left(\bigcap_{i=1}^{6}\left\{X\left(t_{i}\right) \leq k_{i}\right\}, m(0, T) \leq b\right) \\
& =\operatorname{Pr}^{*}\left(\bigcap_{i=1}^{6}\left\{X\left(t_{i}\right) \leq k_{i}\right\}\right)-\operatorname{Pr}^{*}\left(\bigcap_{i=1}^{6}\left\{X\left(t_{i}\right) \leq k_{i}\right\}, m(0, T)>b\right) .
\end{aligned}
$$


The second probability of right-hand side of (A.4) is the same as $P_{7}$ except that drift changes from $r-\sigma^{2} / 2$ into $r+\sigma^{2} / 2$. Thus, we can obtain

$$
\operatorname{Pr}^{*}\left(\bigcap_{i=1}^{6}\left\{X\left(t_{i}\right) \leq k_{i}\right\}\right)-\sum_{J \subset\{1, \ldots, 6\}}(-1)^{|J|} \operatorname{Pr}^{*}\left(\bigcap_{j \in 1}\left\{X\left(t_{i}\right) \leq k_{i}\right\}, m(0, T)>b\right) .
$$

\section{Appendix B: Proof of (3.2) and (3.3) in Lemma 2}

Proof of (3.2): For $b<k_{i}, i=1, \ldots, 6$,

$$
P_{7}=\operatorname{Pr}\left(\bigcap_{i=1}^{6}\left\{b<X\left(t_{i}\right) \leq k_{i}\right\}, m(0, T)>b\right) .
$$

By conditioning on $X\left(t_{i}\right)$, we obtain

$$
\operatorname{Pr}\left(\bigcap_{i=1}^{6}\left\{b<X\left(t_{i}\right) \leq k_{i}\right\}\right) \times \operatorname{Pr}\left(m(0, T)>b \mid \bigcap_{i=1}^{6}\left\{b<X\left(t_{i}\right) \leq k_{i}\right\}\right) .
$$

Since not touching a barrier $b$ contract period and not touching the barrier in all subperiod are equivalent, the second probability of (B.2) can be rewritten as

$$
\operatorname{Pr}\left(\bigcap_{i=1}^{6}\left\{m\left(t_{i-1}, t_{i}\right)>b\right\} \mid \bigcap_{i=1}^{6}\left\{b<X\left(t_{i}\right) \leq k_{i}\right\}\right)=\mathrm{E}\left[\operatorname{Pr}\left(\bigcap_{i=1}^{6}\left\{m\left(t_{i-1}, t_{i}\right)>b\right\} \mid \mathbf{X}\right) \mid \bigcap_{i=1}^{6}\left\{b<X\left(t_{i}\right) \leq k_{i}\right\}\right],
$$

where $\mathbf{X}=\left(X\left(t_{1}\right), X\left(t_{2}\right), \ldots, X\left(t_{6}\right)\right)$. By subtracting $X\left(t_{i}\right)$ on both sides of the inside conditional probability in (B.3), we have

$$
\mathrm{E}\left[\operatorname{Pr}\left(\bigcap_{i=1}^{6}\left\{m\left(t_{i-1}, t_{i}\right)-X\left(t_{i-1}\right)>b-X\left(t_{i-1}\right)\right\} \mid \mathbf{X}\right) \mid \bigcap_{i=1}^{6}\left\{b<X\left(t_{i}\right) \leq k_{i}\right\}\right] .
$$

With independent increment, $m\left(t_{i-1}, t_{i}\right)-X\left(t_{i-1}\right)$ given $\mathbf{X}$ are independent for all $i$, we have

$$
\begin{aligned}
& \mathrm{E}\left[\prod_{i=1}^{6} \operatorname{Pr}\left(m\left(t_{i-1}, t_{i}\right)-X\left(t_{i-1}\right)>b-X\left(t_{i-1}\right) \mid \mathbf{X}\right) \mid \bigcap_{i=1}^{6}\left\{b<X\left(t_{i}\right) \leq k_{i}\right\}\right] \\
& =\mathrm{E}\left[\prod_{i=1}^{6} \operatorname{Pr}\left(m\left(t_{i-1}, t_{i}\right)>b \mid \mathbf{X}\right) \mid \bigcap_{i=1}^{6}\left\{b<X\left(t_{i}\right) \leq k_{i}\right\}\right] \\
& =\mathrm{E}\left[\prod_{i=1}^{6} \operatorname{Pr}\left(m\left(t_{i-1}, t_{i}\right)>b \mid X\left(t_{i-1}\right), X\left(t_{i}\right)\right) \mid \bigcap_{i=1}^{6}\left\{b<X\left(t_{i}\right) \leq k_{i}\right\}\right] .
\end{aligned}
$$

Proof of (3.3): Proof of (3.3) is similar to that of (3.2). For $b<k_{i}, i=1, \ldots, 6$,

$$
P_{8}=\operatorname{Pr}^{*}\left(\bigcap_{i=1}^{6}\left\{X\left(t_{i}\right) \leq k_{i}\right\}, m(0, T) \leq b\right) .
$$


By conditioning on $X\left(t_{i}\right)$, we obtain

$$
\begin{aligned}
& \operatorname{Pr}^{*}\left(\bigcap_{i=1}^{6}\left\{X\left(t_{i}\right) \leq k_{i}\right\}\right) \times \operatorname{Pr}^{*}\left(m(0, T) \leq b \mid \bigcap_{i=1}^{6}\left\{X\left(t_{i}\right) \leq k_{i}\right\}\right) \\
& =\operatorname{Pr}^{*}\left(\bigcap_{i=1}^{6}\left\{X\left(t_{i}\right) \leq k_{i}\right\}\right) \times\left(1-\operatorname{Pr}^{*}\left(m(0, T)>b \mid \bigcap_{i=1}^{6}\left\{X\left(t_{i}\right) \leq k_{i}\right\}\right)\right) .
\end{aligned}
$$

Since not touching a barrier $b$ contract period and not touching the barrier in all subperiod are equivalent, the second probability of (B.7) can be rewritten as

$$
1-\operatorname{Pr}^{*}\left(\bigcap_{i=1}^{6}\left\{m\left(t_{i-1}, t_{i}\right)>b\right\} \mid \bigcap_{i=1}^{6}\left\{X\left(t_{i}\right) \leq k_{i}\right\}\right)=1-\mathrm{E}^{*}\left[\operatorname{Pr}^{*}\left(\bigcap_{i=1}^{6}\left\{m\left(t_{i-1}, t_{i}\right)>b\right\} \mid \mathbf{X}\right) \mid \bigcap_{i=1}^{6}\left\{X\left(t_{i}\right) \leq k_{i}\right\}\right] .
$$

Again, by subtracting $X\left(t_{i}\right)$, we have

$$
\begin{aligned}
& 1-\mathrm{E}^{*}\left[\operatorname{Pr}^{*}\left(\bigcap_{i=1}^{6}\left\{m\left(t_{i-1}, t_{i}\right)-X\left(t_{i-1}\right)>b-X\left(t_{i-1}\right)\right\} \mid \mathbf{X}\right) \mid \bigcap_{i=1}^{6}\left\{X\left(t_{i}\right) \leq k_{i}\right\}\right] \\
& =1-\mathrm{E}^{*}\left[\prod_{i=1}^{6} \operatorname{Pr}^{*}\left(m\left(t_{i-1}, t_{i}\right)-X\left(t_{i-1}\right)>b-X\left(t_{i-1}\right) \mid \mathbf{X}\right) \mid \bigcap_{i=1}^{6}\left\{X\left(t_{i}\right) \leq k_{i}\right\}\right] \\
& =1-\mathrm{E}^{*}\left[\prod_{i=1}^{6} \operatorname{Pr}^{*}\left(m\left(t_{i-1}, t_{i}\right)>b \mid X\left(t_{i-1}\right), X\left(t_{i}\right)\right) \mid \bigcap_{i=1}^{6}\left\{X\left(t_{i}\right) \leq k_{i}\right\}\right] .
\end{aligned}
$$

\section{Appendix C: BB algorithm for pricing ELS}

\section{Step 1 Define the input parameters}

Set the number of scenarios $N$, autocallable date and maturity $\left(t_{1}, \ldots, t_{6}\right)$, autocall threshold $\left(k_{1}, \ldots, k_{6}\right)$, KI barrier $b$, the risk-free rate $r$, volatility $\sigma$, coupon rate $C$, and initial underlying index $S(0)$. Let $\mu_{1}=r-\sigma^{2} / 2, \mu_{2}=r+\sigma^{2} / 2$ and correlation coefficient matrix $\rho$ where components of the matrix $\rho_{i j}=\sqrt{\min \left(t_{i}, t_{j}\right) / \max \left(t_{i}, t_{j}\right)}$.

\section{Step 2 Compute $P_{1}$ to $P_{6}$}

$P_{1}$ to $P_{6}$ can be easily computed by statistical packages.

$P_{1}=\operatorname{Pr}\left(X\left(t_{1}\right)>k_{1}\right)$,

$P_{2}=\operatorname{Pr}\left(X\left(t_{1}\right)<k_{1}, X\left(t_{2}\right)>k_{2}\right)$,

:

$P_{6}=\operatorname{Pr}\left(X\left(t_{1}\right)<k_{1}, \ldots, X\left(t_{6}\right)>k_{6}\right)$. 


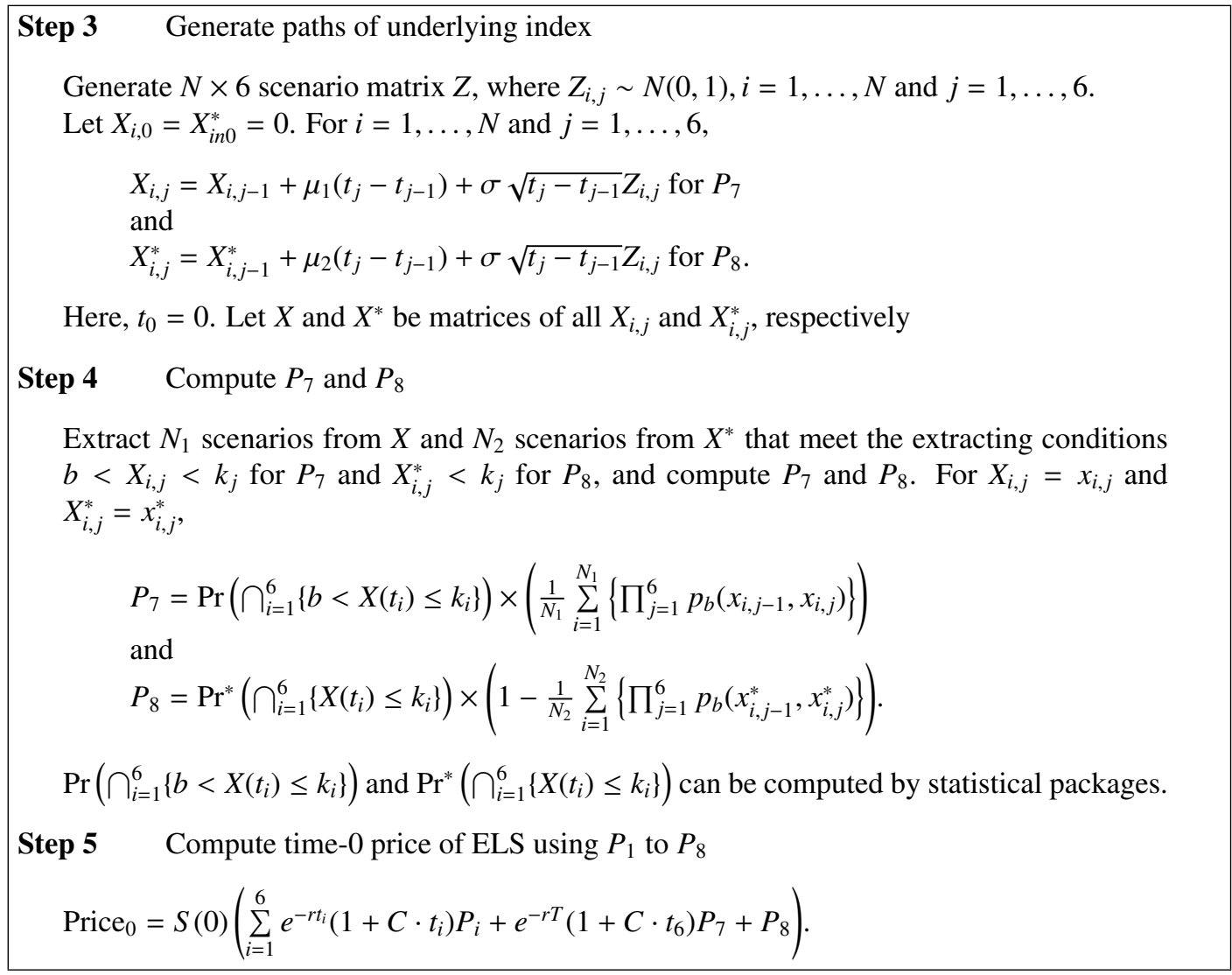

\section{References}

Deng G, Husson T, and McCann C (2014). Valuation of structured products, The Journal of Alternative Investments, 16, 71-87.

Deng G, Mallett J, and McCann C (2016). Modeling autocallable structured products. In Derivatives and Hedge Funds (pp. 323-334), Palgrave Macmillan, United Kingdom.

Gerber HU and Shiu ESW (1994). Option pricing by esscher transforms, Transactions of the Society of Actuaries, 46, 99-191.

Gobet E (2009). Advanced Monte Carlo methods for barrier and related exotic options, In Handbook of Numerical Analysis, 15, 497-528.

Guillaume T (2015). Autocallable structured products, The Journal of Derivatives, 22, 73-94.

Huh J and Kolkiewicz A (2008). Computation of multivariate barrier crossing probability and its applications in credit risk models, North American Actuarial Journal, 12, 263-291.

Jeong DR, Wee IS, and Kim JS (2010). An operator splitting method for pricing the ELS option, Journal of the Korean Society for Industrial and Applied Mathematics, 14, 175-187.

Jang H, Kim S, Han J, et al. (2019). Fast Monte Carlo simulation for pricing equity-linked securities, Computational Economics, 56, 1-18.

Kim KK and Lim DY (2019). A recursive method for static replication of autocallable structured products, Quantitative Finance, 19, 647-661. 
Kim Y, Bae HO, and Roh HS (2011). FDM Algorithm for pricing of ELS with exit probability, Journal of Derivatives and Quantitative Studies, 19, 427-446.

Korn R, Korn E, and Kroisandt G (2010). Monte Carlo methods and models in finance and insurance, CRC Press, United States

Lee C, Lyu J, Park E, Lee W, Kim S, Jeong D, and Kim J (2020). Super-fast computation for the three-asset equity-linked securities using the finite difference method, Mathematics, 8, 307.

Lee H, Ahn S, and Ko B (2019). Generalizing the reflection principle of Brownian motion, and closedform pricing of barrier options and autocallable investments, The North American Journal of Economics and Finance, 50, 101014.

Received December 10, 2020; Revised January 26, 2021; Accepted February 20, 2021 
